\title{
Insect antifeedant potent unsaturated 1,3-oxazine-2-amines
}

\author{
G. Thirunarayanan ${ }^{1, \star}$, V. Renuka ${ }^{2}$, K. G. Sekar ${ }^{2}$, K. Lakshmanan ${ }^{2}$, K. Anbarasu ${ }^{3}$ \\ ${ }^{1}$ Department of Chemistry, Annamalai University, Annamalainagar - 608002, India \\ ${ }^{2}$ Department of Chemistry, National College, Tiruchirappalli - 620001, India \\ ${ }^{3}$ Department of Chemistry, TRP Engineering College (SRM), Irungalur - 621105, India \\ E-mail address: drgtnarayanan@gmail.com
}

\begin{abstract}
Insect antifeedant activities of some halo substituted aryl 1,3-oxazine-2-amines have been measured using $4^{\text {th }}$ instar larvae Achoea Janata $L$ by castor leaf discs-Dethler's method. The highly halo substituted oxazine amines have shown good insect antifeedant activities. The 1,3-oxazine amines have been synthesised by greener method by solvent-free cyclization of aryl enones with urea under microwave irradiation. The yields of the oxazines were more than $80 \%$. The synthesised oxazines were characterized by their physical constants, analytical and spectroscopic data.
\end{abstract}

Keywords: Insect antifeedant activity; $4^{\text {th }}$ instar larvae Achoea Janata L; Aryl 1,3-oxazine; Castor leaf discs; Green synthesis; IR and NMR spectra

\section{INTRODUCTION}

Numerous organic compounds used as agrochemicals such as halo ketones, unsaturated halo ketones [1], acyl halides [2], alkaloids [3], many naturally occurring compounds from plant extracts [4,5] and $\omega$-substituted halo compounds [2]. This agrochemical possess many biological activities such as antimicrobial [6], antioxidant [7], fumigant activity [8], insecticides [9], weedicides [10], larvacidal [11] and insect antifeedants [12,13] due to the presence of alkenes, carbonyl, halogens and some polar groups in their structural moiety. The insect antifeedant activity is important in agricultural-agronomy fields. Due to feeding of leafs by the insects; the economic crises will occur in this field by low quality, recovery and finally the valuable plants will be lost. Therefore the insect antifeedant agents are important for protection of our plants. Many methods available for measuring this insect antifeedant activity such as, Boll Weevil antifeedant bioassay [14], castor leaf disc bioassay [15] and sweet potato leafs [16], Similarly many insects employed for evaluate the activity such as, $4^{\text {th }}$ instar larvae Achoe Janta L [17], and common cut worms [18]. Recently Thirunarayanan et al., [19,23-27] have studied the insect antifeedant activities of some synthetic chalcones and acyl compounds. Nalwar, et al., [20] also studied the insect antifeedant activities with cotton leaf 
by in-vitro method. Dasharathi et al., [21] have studied the insect antifeedant activities of halogenated phenyl naphthyl chalcones. Within the above view, there is no report available for the synthesis and the study of insect antifeedant activities of halo oxazine derivatives. Therefore the author have taken efforts to synthesize of some halogenated oxazine derivatives and study the insect antifeedant activities using $4^{\text {th }}$ instar larvae Achoea Janata L by castor leaf discs-Dethler's [22] method.

\section{EXPERIMENTAL}

\section{1. General}

All chemicals used in this study were procure from Sigma-Aldrich Chemical company. Mettler FP51 melting point apparatus was used for determining the melting point of all synthesized oxazine-2-amines in open glass capillaries and are uncorrected. The AVATAR300 Fourier transform spectrophotometer was used for recording infrared spectra $(\mathrm{KBr}, 4000$ $400 \mathrm{~cm}^{-1}$ ) of all oxazines in $\mathrm{KBr}$ disc. The Bruker AV400 type NMR spectrometer was utilized for recording NMR spectra of all oxazines, operating at $400 \mathrm{MHz}$ for ${ }^{1} \mathrm{H}$ and 100 $\mathrm{MHz}$ for ${ }^{13} \mathrm{C}$ spectra in $\mathrm{CDCl}_{3}$ solvent using TMS as internal standard. Mass spectra of all synthesised oxazines were recorded on SHIMADZU mass spectrometer using chemical ionization technique.

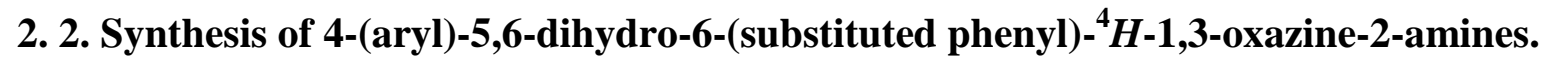

An appropriate equi-molar quantities of chalcones $(2 \mathrm{mmol})$, urea $(2 \mathrm{mmol})$ and $0.2 \mathrm{~g}$ of $\mathrm{KF} / \mathrm{Al}_{2} \mathrm{O}_{3}$ were taken in a $50 \mathrm{~mL}$ beaker, closed with the lid. This mixture was subjected to microwave irradiation for 2-4 minutes at 650W (Scheme 1) (Samsung, Microwave Oven, 100-700 W). After completion of the reaction, dichloromethane $(20 \mathrm{~mL})$ was added, followed by simple filtration. The solution was concentrated and purified by re-crystallization. The synthesized oxazines were characterized by their physical constants, IR, ${ }^{1} \mathrm{H}$ and ${ }^{13} \mathrm{C} N M R$ and Mass spectral data. The analytical, physical constants and mass fragments $(\mathrm{m} / \mathrm{z})$ data are presented in Table 1.<smiles>[R]C=C(C)C([R])=O</smiles>

Scheme 1. Synthesis of 4-aryl-5,6-dihydro-6-(substituted phenyl)- ${ }^{4} H$-1,3-oxazine-2-amines $\mathrm{KF} / \mathrm{Al}_{2} \mathrm{O}_{3}$ catalyzed cyclization of aryl chalcones and urea under microwave irradiation.

The infrared and NMR spectral data of the synthesised oxazines are summarized below.

1. 4-Phenyl-5,6-dihydro-6-(4-bromophenyl)- ${ }^{4} H$-1,3-oxazine-2-amines: FTIR (KBr): 3535 $(\mathrm{NH}), 1596(\mathrm{C}=\mathrm{N}), 1210(\mathrm{C}-\mathrm{O}-\mathrm{C}) \mathrm{cm}^{-1} .{ }^{1} \mathrm{H} \mathrm{NMR}\left(\mathrm{CDCl}_{3}-\mathrm{d}_{6}, \mathrm{TMS}\right) \delta: 2.323\left(\mathrm{~s}, 1 \mathrm{H}, \mathrm{NH}_{2}\right)$, 2.634(dd, $\left.1 \mathrm{H}, \mathrm{H}_{4}\right), 2.413\left(\mathrm{dd}, 1 \mathrm{H}, \mathrm{H}_{5}\right), 2.222\left(\mathrm{dd}, 1 \mathrm{H}, \mathrm{H}_{5^{\prime}}\right), 4.252\left(\mathrm{dd}, 1 \mathrm{H}, \mathrm{H}_{6}\right), 6.543-7.367(\mathrm{~m}$, 
9H, Ar-H) ppm.; ${ }^{13} \mathrm{C} \mathrm{NMR}\left(\mathrm{CDCl}_{3}-\mathrm{d}_{6}, \mathrm{TMS}\right) \delta:$ 165.47( $\left.\mathrm{C}_{2}\right), 52.65\left(\mathrm{C}_{4}\right), 47.78\left(\mathrm{C}_{5}\right), 65.78\left(\mathrm{C}_{6}\right)$, 125.47-142.89(Ar-C)ppm.

2. 4-Phenyl-5,6-dihydro-6-(4-chlorophenyl)- ${ }^{4} \boldsymbol{H}$-1,3-oxazine-2-amines: FTIR (KBr): 3538 $(\mathrm{NH}), 1589(\mathrm{C}=\mathrm{N}), 1223(\mathrm{C}-\mathrm{O}-\mathrm{C}) \mathrm{cm}^{-1} .{ }^{1} \mathrm{H} \mathrm{NMR}\left(\mathrm{CDCl}_{3}-\mathrm{d}_{6}, \mathrm{TMS}\right) \delta: 2.313\left(\mathrm{~s}, 1 \mathrm{H}, \mathrm{NH}_{2}\right)$, 2.645(dd, $\left.1 \mathrm{H}, \mathrm{H}_{4}\right), 2.423\left(\mathrm{dd}, 1 \mathrm{H}, \mathrm{H}_{5}\right), 2.219\left(\mathrm{dd}, 1 \mathrm{H}, \mathrm{H}_{5^{\prime}}\right), 4.265\left(\mathrm{dd}, 1 \mathrm{H}, \mathrm{H}_{6}\right), 6.534-7.387(\mathrm{~m}$, 9H, Ar-H) ppm.; ${ }^{13} \mathrm{C} \mathrm{NMR}\left(\mathrm{CDCl}_{3}-\mathrm{d}_{6}, \mathrm{TMS}\right) \delta$ : 165.32( $\left.\left.\mathrm{C}_{2}\right), 52.87\left(\mathrm{C}_{4}\right), 47.98\left(\mathrm{C}_{5}\right), 65.47 \mathrm{C}_{6}\right)$, 125.54-142.67(Ar-C)ppm.

3. 4-Chlorohenyl-5,6-dihydro-6-(4-bromophenyl)- ${ }^{4} H$-1,3-oxazine-2-amines: FTIR (KBr): $3547(\mathrm{NH}), 1598(\mathrm{C}=\mathrm{N}), 1220(\mathrm{C}-\mathrm{O}-\mathrm{C}) \mathrm{cm}^{-1} .{ }^{1} \mathrm{H}$ NMR $\left(\mathrm{CDCl}_{3}-\mathrm{d}_{6}, \mathrm{TMS}\right) \delta: 2.319\left(\mathrm{~s}, 1 \mathrm{H}, \mathrm{NH}_{2}\right)$, 2.625(dd, $\left.1 \mathrm{H}, \mathrm{H}_{4}\right), 2.467\left(\mathrm{dd}, 1 \mathrm{H}, \mathrm{H}_{5}\right), 2.249\left(\mathrm{dd}, 1 \mathrm{H}, \mathrm{H}_{5^{\prime}}\right), 4.267\left(\mathrm{dd}, 1 \mathrm{H}, \mathrm{H}_{6}\right), 6.545-7.398(\mathrm{~m}$, $8 \mathrm{H}, \mathrm{Ar}-\mathrm{H}) \mathrm{ppm} .{ }^{13} \mathrm{C} \mathrm{NMR}\left(\mathrm{CDCl}_{3}-\mathrm{d}_{6}, \mathrm{TMS}\right) \delta$ : $165.90\left(\mathrm{C}_{2}\right), 52.78\left(\mathrm{C}_{4}\right), 47.57\left(\mathrm{C}_{5}\right), 65.78\left(\mathrm{C}_{6}\right)$, 125.34-142.80(Ar-C)ppm.

4. 4-Chlorohenyl-5,6-dihydro-6-(4-chlorophenyl)- ${ }^{4} H$-1,3-oxazine-2-amine: FTIR (KBr): 3555(NH), 1593(C=N), 1216(C-O-C) $\mathrm{cm}^{-1} .{ }^{1} \mathrm{H}$ NMR $\left(\mathrm{CDCl}_{3}-\mathrm{d}_{6}, \mathrm{TMS}\right) \delta: 2.324\left(\mathrm{~s}, 1 \mathrm{H}, \mathrm{NH}_{2}\right)$, 2.365(dd, $\left.1 \mathrm{H}, \mathrm{H}_{4}\right), 2.476\left(\mathrm{dd}, 1 \mathrm{H}, \mathrm{H}_{5}\right), 2.246\left(\mathrm{dd}, 1 \mathrm{H}, \mathrm{H}_{5^{\prime}}\right), 4.291\left(\mathrm{dd}, 1 \mathrm{H}, \mathrm{H}_{6}\right), 6.576-7.392(\mathrm{~m}$, $8 \mathrm{H}, \mathrm{Ar}-\mathrm{H}) \mathrm{ppm} .{ }^{13} \mathrm{C} \mathrm{NMR}\left(\mathrm{CDCl}_{3}-\mathrm{d}_{6}, \mathrm{TMS}\right) \delta$ : 165.56( $\left(\mathrm{C}_{2}\right), 52.89\left(\mathrm{C}_{4}\right), 47.47\left(\mathrm{C}_{5}\right), 65.70\left(\mathrm{C}_{6}\right)$, 125.34-142.78(Ar-C)ppm.

Table 1. Analytical, physical constants, yield and mass fragment of 4-aryl-5,6-dihydro-6(substituted phenyl)- ${ }^{4} H-1,3$-oxazine-2-amines.

\begin{tabular}{|c|c|c|c|c|c|c|}
\hline Entry & $\mathrm{R}$ & $\mathrm{R}^{\prime}$ & M. W. & $\begin{array}{c}\text { Yield } \\
(\%)\end{array}$ & $\begin{array}{l}\text { m.p. } \\
\left({ }^{\circ} \mathrm{C}\right)\end{array}$ & $\operatorname{Mass}(\mathrm{m} / \mathrm{z})$ \\
\hline 1 & & & 332 & 87 & $\begin{array}{l}m \\
\stackrel{m}{1} \\
\stackrel{n}{n}\end{array}$ & $\begin{array}{c}332\left[\mathrm{M}^{+}\right], 334 \\
{\left[\mathrm{M}^{2+}\right], 314} \\
272,237,215 \\
175,154,79,77 \\
58,43,42.16 \\
\end{array}$ \\
\hline 2 & & & 288 & 85 & $\begin{array}{l}\text { ป̃ } \\
\text { ป் }\end{array}$ & $\begin{array}{c}286\left[\mathrm{M}^{+}\right], 288 \\
{\left[\mathrm{M}^{2+}\right], 270,266,} \\
251,175,160, \\
111,107,99,84 \\
77,43,42,35,16\end{array}$ \\
\hline 3 & $\mathrm{Cl}$ & & 365 & 86 & $\begin{array}{l}\stackrel{\infty}{\sim} \\
\stackrel{1}{\simeq}\end{array}$ & $\begin{array}{c}365\left[\mathrm{M}^{+}\right], 367 \\
{\left[\mathrm{M}^{2+}\right], 369\left[\mathrm{M}^{4+}\right]} \\
374,285,252, \\
209,154,99,79, \\
54,16,\end{array}$ \\
\hline 4 & $\mathrm{Cl}-$ & & 322 & 85 & $\begin{array}{l}\Xi \\
\stackrel{\Xi}{n} \\
=\end{array}$ & $\begin{array}{c}322\left[\mathrm{M}^{+}\right], 324 \\
{\left[\mathrm{M}^{2+}\right], 377\left[\mathrm{M}^{4+}\right]} \\
304,209,111, \\
99.77,35,16\end{array}$ \\
\hline
\end{tabular}




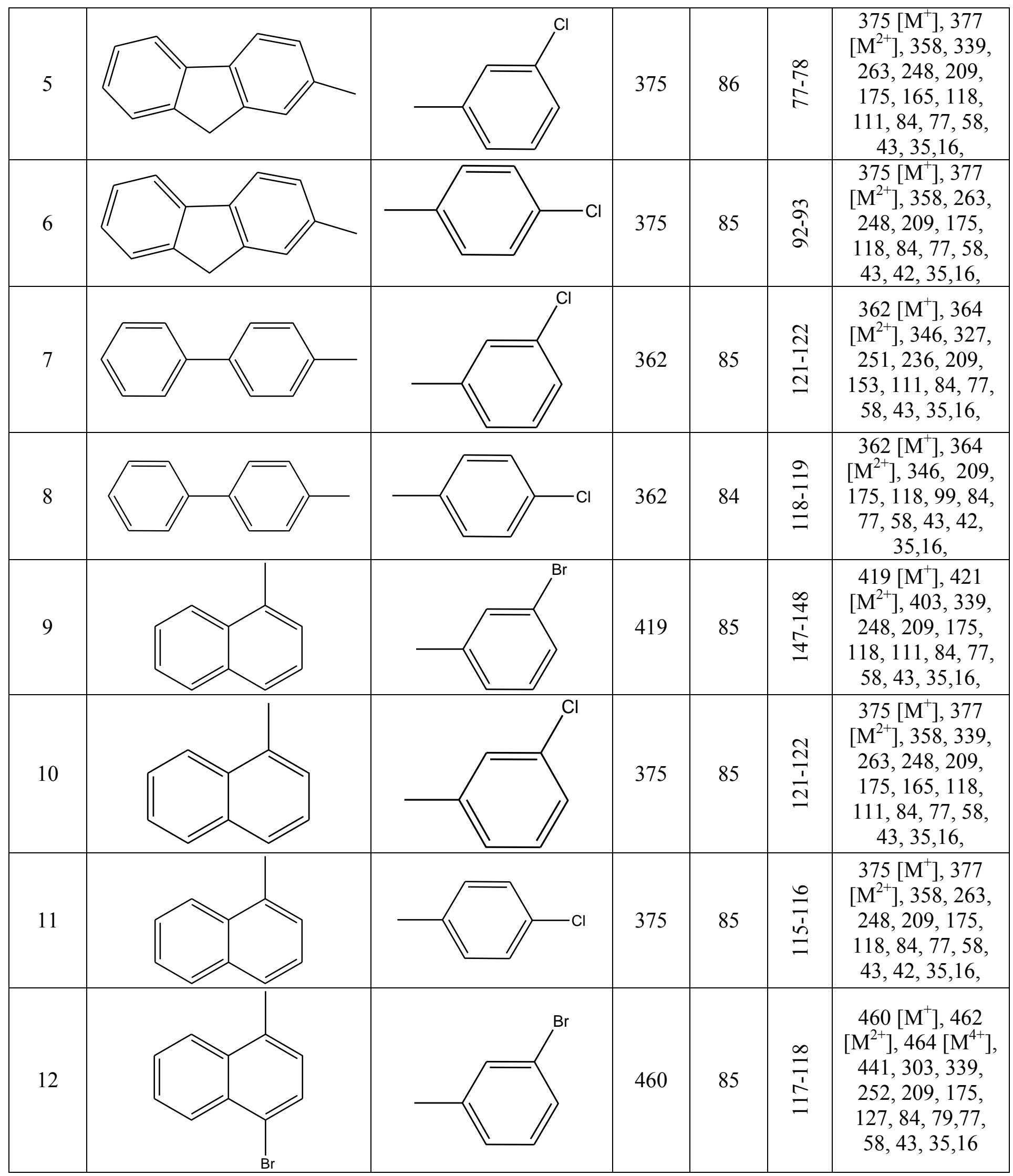




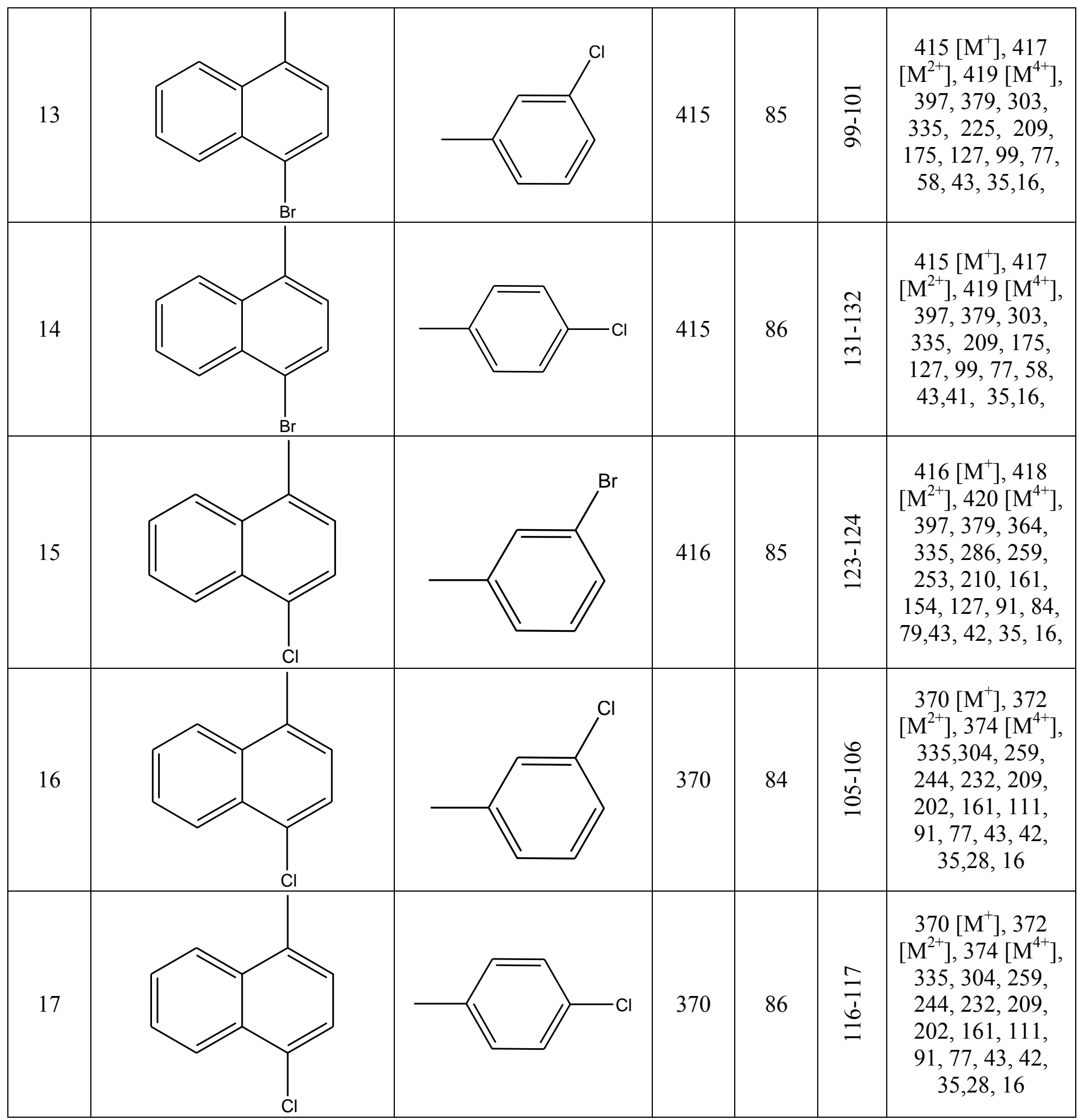




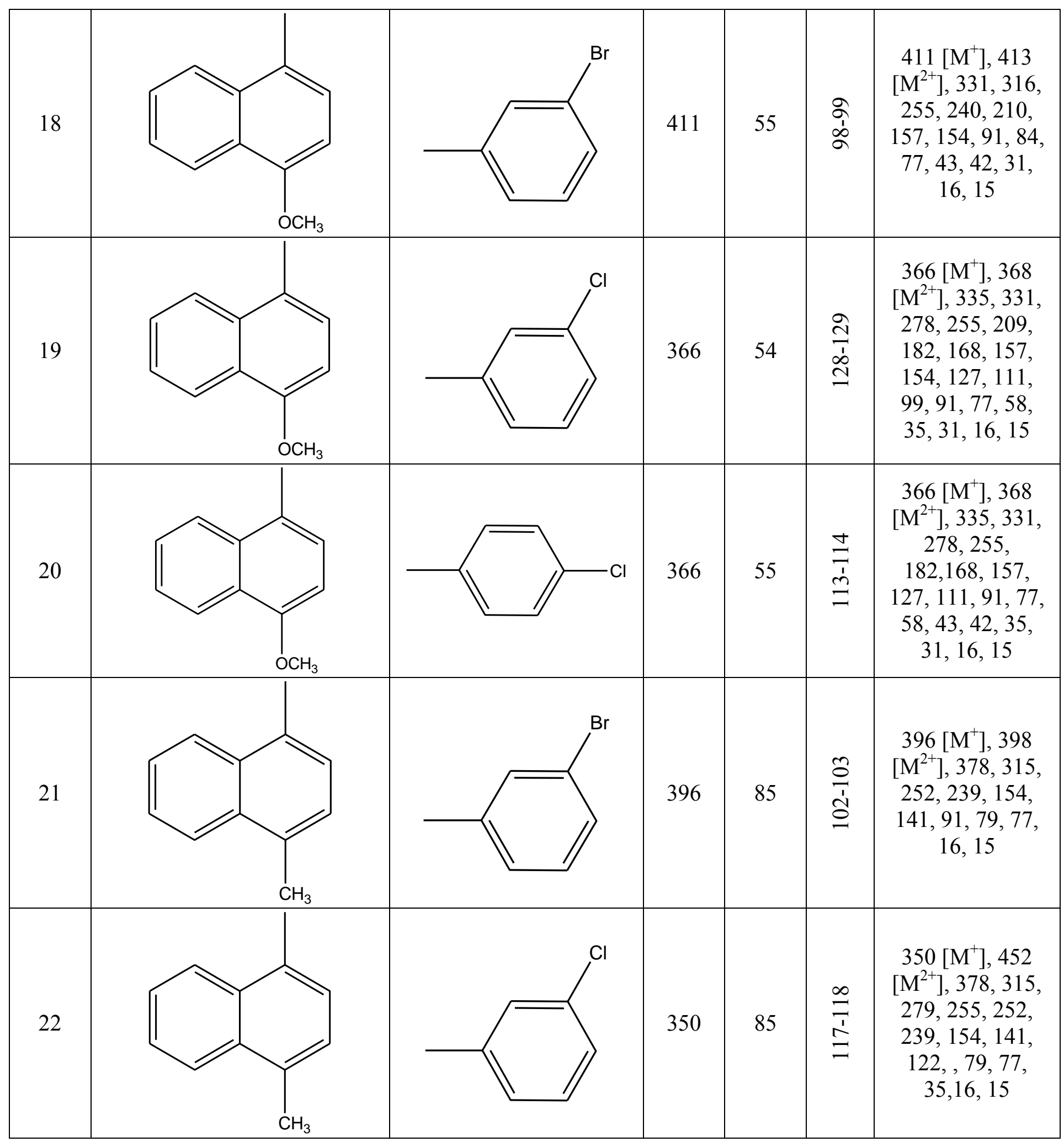




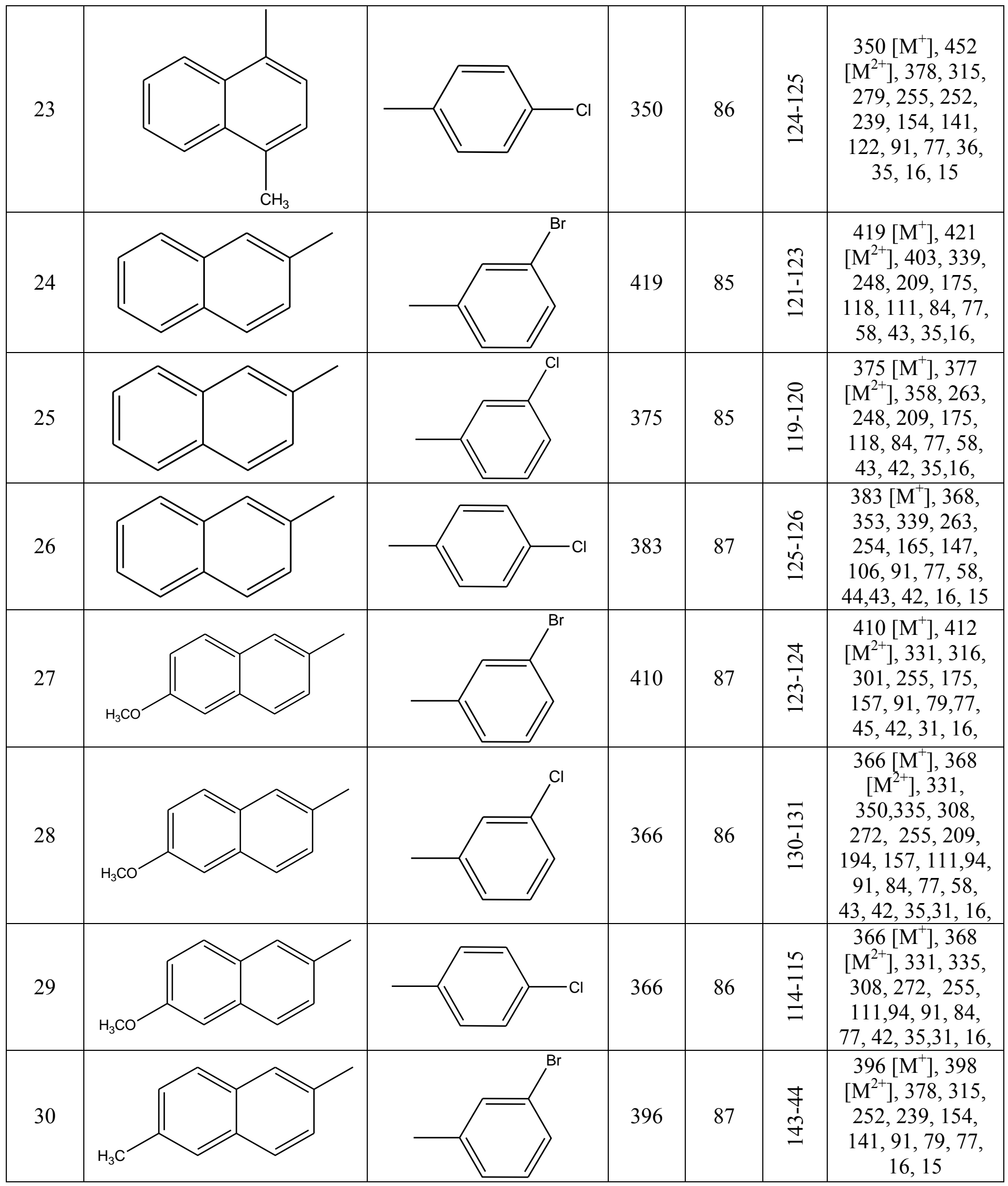




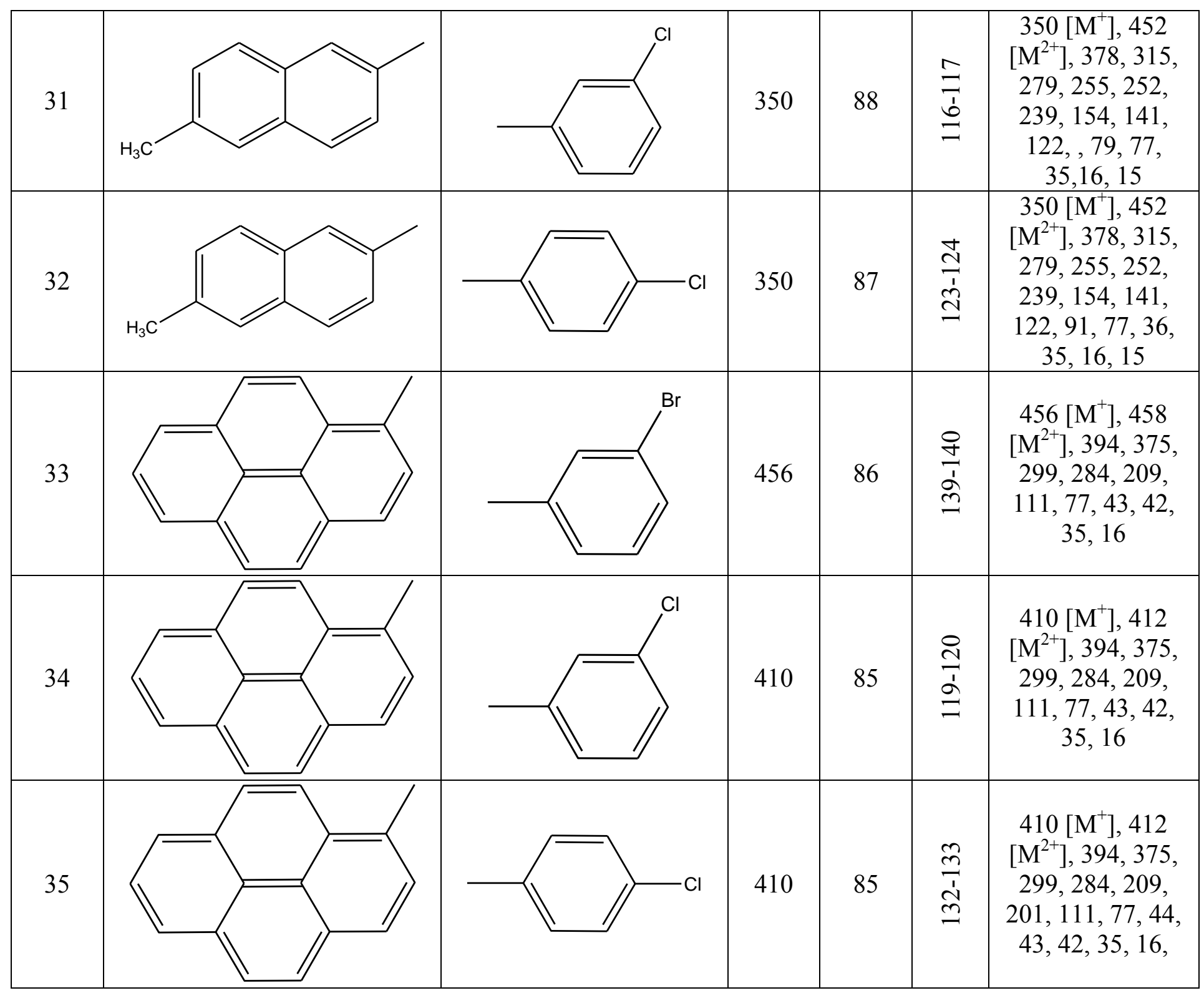

5. 4-(9H-Fluorene-2-yl)-5,6-dihydro-6-(3-phenylphenyl)- ${ }^{4} H$-1,3-oxazine-2-amine: FTIR $(\mathrm{KBr}): 3545(\mathrm{NH}), 1593(\mathrm{C}=\mathrm{N}), 1216(\mathrm{C}-\mathrm{O}-\mathrm{C}) \mathrm{cm}^{-1} .{ }^{1} \mathrm{H}$ NMR $\left(\mathrm{CDCl}_{3}-\mathrm{d}_{6}, \mathrm{TMS}\right) \delta: 2.254(\mathrm{~s}, 1 \mathrm{H}$, $\left.\mathrm{NH}_{2}\right), 2.215\left(\mathrm{dd}, 1 \mathrm{H}, \mathrm{H}_{4}\right), 2.264\left(\mathrm{dd}, 1 \mathrm{H}, \mathrm{H}_{5}\right), 2.031\left(\mathrm{dd}, 1 \mathrm{H}, \mathrm{H}_{5^{\prime}}\right), 4.625\left(\mathrm{dd}, 1 \mathrm{H}, \mathrm{H}_{6}\right), 6.715-$ 7.775(m, 11H, Ar-H) ppm.; ${ }^{13} \mathrm{C}$ NMR $\left(\mathrm{CDCl}_{3}-\mathrm{d}_{6}, \mathrm{TMS}\right) \delta: 164.89\left(\mathrm{C}_{2}\right), 52.31\left(\mathrm{C}_{4}\right), 47.26\left(\mathrm{C}_{5}\right)$, $67.29\left(\mathrm{C}_{6}\right), 47.26\left(\mathrm{OCH}_{3}\right)$, 121.25-138.32 (Ar-C)ppm.

6. 4-(9H-Fluorene-2-yl)-5,6-dihydro-6-(4-chlorophenyl)- ${ }^{4} H$-1,3-oxazine-2-amine: FTIR $(\mathrm{KBr}): 3548(\mathrm{NH}), 1602(\mathrm{C}=\mathrm{N}), 1215(\mathrm{C}-\mathrm{O}-\mathrm{C}) \mathrm{cm}^{-1} .{ }^{1} \mathrm{H} \mathrm{NMR}\left(\mathrm{CDCl}_{3}-\mathrm{d}_{6}, \mathrm{TMS}\right) \delta: 2.156(\mathrm{~s}, 1 \mathrm{H}$, $\left.\mathrm{NH}_{2}\right), 2.251\left(\mathrm{dd}, 1 \mathrm{H}, \mathrm{H}_{4}\right), 2.268\left(\mathrm{dd}, 1 \mathrm{H}, \mathrm{H}_{5}\right), 2.054\left(\mathrm{dd}, 1 \mathrm{H}, \mathrm{H}_{5^{\prime}}\right), 4.698\left(\mathrm{dd}, 1 \mathrm{H}, \mathrm{H}_{6}\right), 6.853-$ 7.895(m, 11H, Ar-H) ppm.; ${ }^{13} \mathrm{C} \mathrm{NMR}\left(\mathrm{CDCl}_{3}-\mathrm{d}_{6}, \mathrm{TMS}\right) \delta: 165.02\left(\mathrm{C}_{2}\right), 52.16\left(\mathrm{C}_{4}\right), 47.29\left(\mathrm{C}_{5}\right)$, $67.28\left(\mathrm{C}_{6}\right), 121.35-139.35(\mathrm{Ar}-\mathrm{C}) \mathrm{ppm}$.

7. 4-(4-Biphenyl)-5,6-dihydro-6-(3-chlorophenyl)- ${ }^{4} H$-1,3-oxazine-2-amine: FTIR (KBr): 3552(NH), 1593(C=N), 1221(C-O-C) $\mathrm{cm}^{-1} .{ }^{1} \mathrm{H}$ NMR $\left(\mathrm{CDCl}_{3}-\mathrm{d}_{6}, \mathrm{TMS}\right) \delta: 2.242\left(\mathrm{~s}, 1 \mathrm{H}, \mathrm{NH}_{2}\right)$, 2.217(dd, $\left.1 \mathrm{H}, \mathrm{H}_{4}\right), 2.254\left(\mathrm{dd}, 1 \mathrm{H}, \mathrm{H}_{5}\right), 2.024\left(\mathrm{dd}, 1 \mathrm{H}, \mathrm{H}_{5^{\prime}}\right), 4.635\left(\mathrm{dd}, 1 \mathrm{H}, \mathrm{H}_{6}\right), 6.714-7.775(\mathrm{~m}$, 
11H, Ar-H) ppm.; ${ }^{13} \mathrm{C} \mathrm{NMR}\left(\mathrm{CDCl}_{3}-\mathrm{d}_{6}, \mathrm{TMS}\right) \delta: 164.89\left(\mathrm{C}_{2}\right), 52.31\left(\mathrm{C}_{4}\right), 47.36\left(\mathrm{C}_{5}\right), 67.25$ $\left(\mathrm{C}_{6}\right), 121.35-138.19$ (Ar-C)ppm.

8. 4-(4-Biphenyl)-5,6-dihydro-6-(4-chlorophenyl)- ${ }^{4} H$-1,3-oxazine-2-amine: FTIR (KBr): 3555(NH), $1610(\mathrm{C}=\mathrm{N}), 1212(\mathrm{C}-\mathrm{O}-\mathrm{C}) \mathrm{cm}^{-1} .{ }^{1} \mathrm{H}$ NMR $\left(\mathrm{CDCl}_{3}-\mathrm{d}_{6}, \mathrm{TMS}\right) \delta: 2.125\left(\mathrm{~s}, 1 \mathrm{H}, \mathrm{NH}_{2}\right)$, 2.274(dd, $\left.1 \mathrm{H}, \mathrm{H}_{4}\right), 2.277\left(\mathrm{dd}, 1 \mathrm{H}, \mathrm{H}_{5}\right), 2.055\left(\mathrm{dd}, 1 \mathrm{H}, \mathrm{H}_{5^{\prime}}\right), 4.687\left(\mathrm{dd}, 1 \mathrm{H}, \mathrm{H}_{6}\right), 6.851-7.894(\mathrm{~m}$, $11 \mathrm{H}, \mathrm{Ar}-\mathrm{H}) \mathrm{ppm} . ;{ }^{13} \mathrm{C} \mathrm{NMR}\left(\mathrm{CDCl}_{3}-\mathrm{d}_{6}, \mathrm{TMS}\right) \delta$ : $165.22\left(\mathrm{C}_{2}\right), 52.36\left(\mathrm{C}_{4}\right), 47.36\left(\mathrm{C}_{5}\right)$, 67.28( $\left.\mathrm{C}_{6}\right), 121.35-139.35$ (Ar-C)ppm.

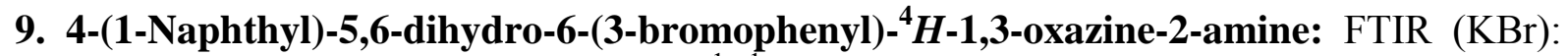
$3548(\mathrm{NH}), 1586(\mathrm{C}=\mathrm{N}), 1226(\mathrm{C}-\mathrm{O}-\mathrm{C}) \mathrm{cm}^{-1} .{ }^{1} \mathrm{H}$ NMR $\left(\mathrm{CDCl}_{3}-\mathrm{d}_{6}, \mathrm{TMS}\right) \delta: 2.234\left(\mathrm{~s}, 1 \mathrm{H}, \mathrm{NH}_{2}\right)$, 2.214(dd, $\left.1 \mathrm{H}, \mathrm{H}_{4}\right), 2.256\left(\mathrm{dd}, 1 \mathrm{H}, \mathrm{H}_{5}\right), 2.028\left(\mathrm{dd}, 1 \mathrm{H}, \mathrm{H}_{5^{\prime}}\right), 4.643\left(\mathrm{dd}, 1 \mathrm{H}, \mathrm{H}_{6}\right), 6.723-7.767(\mathrm{~m}$, $11 \mathrm{H}, \mathrm{Ar}-\mathrm{H}) \mathrm{ppm} ;{ }^{13} \mathrm{C} \mathrm{NMR}\left(\mathrm{CDCl}_{3}-\mathrm{d}_{6}, \mathrm{TMS}\right) \delta$ : $164.37\left(\mathrm{C}_{2}\right), 52.36\left(\mathrm{C}_{4}\right), 47.34\left(\mathrm{C}_{5}\right)$, 66.25( $\left.\mathrm{C}_{6}\right), 121.26-139.98($ Ar-C)ppm.

10. 4-(1-Naphthyl)-5,6-dihydro-6-(3-chlorophenyl)- ${ }^{4} H$-1,3-oxazine-2-amine: FTIR (KBr): 3545(NH), $1612(\mathrm{C}=\mathrm{N}), 1223(\mathrm{C}-\mathrm{O}-\mathrm{C}) \mathrm{cm}^{-1} .{ }^{1} \mathrm{H}$ NMR $\left(\mathrm{CDCl}_{3}-\mathrm{d}_{6}, \mathrm{TMS}\right) \delta: 2.243\left(\mathrm{~s}, 1 \mathrm{H}, \mathrm{NH}_{2}\right)$, 2.212(dd, $\left.1 \mathrm{H}, \mathrm{H}_{4}\right), 2.256\left(\mathrm{dd}, 1 \mathrm{H}, \mathrm{H}_{5}\right), 2.026\left(\mathrm{dd}, 1 \mathrm{H}, \mathrm{H}_{5^{\prime}}\right), 4.634\left(\mathrm{dd}, 1 \mathrm{H}, \mathrm{H}_{6}\right), 6.722-7.789(\mathrm{~m}$, $11 \mathrm{H}, \mathrm{Ar}-\mathrm{H}) \mathrm{ppm} . ;{ }^{13} \mathrm{C} \mathrm{NMR}\left(\mathrm{CDCl}_{3}-\mathrm{d}_{6}, \mathrm{TMS}\right) \delta: 164.37\left(\mathrm{C}_{2}\right), 52.80\left(\mathrm{C}_{4}\right), 47.22\left(\mathrm{C}_{5}\right)$, 66.44( $\left.\mathrm{C}_{6}\right), 21.34-139.38(\mathrm{Ar}-\mathrm{C}) \mathrm{ppm}$.

11. 4-(1-Naphthyl)-5,6-dihydro-6-(4-chlorophenyl)- ${ }^{4} H$-1,3-oxazine-2-amine: FTIR (KBr): $3545(\mathrm{NH}), 1608(\mathrm{C}=\mathrm{N}), 1212(\mathrm{C}-\mathrm{O}-\mathrm{C}) \mathrm{cm}^{-1} .{ }^{1} \mathrm{H}$ NMR $\left(\mathrm{CDCl}_{3}-\mathrm{d}_{6}, \mathrm{TMS}\right) \delta: 2.153\left(\mathrm{~s}, 1 \mathrm{H}, \mathrm{NH}_{2}\right)$, 2.255(dd, $\left.1 \mathrm{H}, \mathrm{H}_{4}\right), 2.260\left(\mathrm{dd}, 1 \mathrm{H}, \mathrm{H}_{5}\right), 2.053\left(\mathrm{dd}, 1 \mathrm{H}, \mathrm{H}_{5^{\prime}}\right), 4.697\left(\mathrm{dd}, 1 \mathrm{H}, \mathrm{H}_{6}\right), 6.851-7.894(\mathrm{~m}$, $11 \mathrm{H}, \mathrm{Ar}-\mathrm{H}) \mathrm{ppm} . ;{ }^{13} \mathrm{C} \mathrm{NMR}\left(\mathrm{CDCl}_{3}-\mathrm{d}_{6}, \mathrm{TMS}\right) \delta$ : $165.18\left(\mathrm{C}_{2}\right), 52.11\left(\mathrm{C}_{4}\right), 47.22\left(\mathrm{C}_{5}\right)$, 67.29( $\left.\mathrm{C}_{6}\right), 121.25-138.32(\mathrm{Ar}-\mathrm{C}) \mathrm{ppm}$.

12. 4-(4-Bromo-1-naphthyl)-5,6-dihydro-6-(3-bromophenyl) $-{ }^{4} H$-1,3-oxazine-2-amine: FTIR (KBr): 3552(NH), 1623(C=N), $1209(\mathrm{C}-\mathrm{O}-\mathrm{C}) \mathrm{cm}^{-1} .{ }^{1} \mathrm{H}$ NMR $\left(\mathrm{CDCl}_{3}-\mathrm{d}_{6}, \mathrm{TMS}\right) \delta: 2.221$ $\left.\left(\mathrm{s}, 1 \mathrm{H}, \mathrm{NH}_{2}\right), 2.314\left(\mathrm{dd}, 1 \mathrm{H}, \mathrm{H}_{4}\right), 2.22 \mathrm{dd}, 1 \mathrm{H}, \mathrm{H}_{5}\right), 2.236\left(\mathrm{dd}, 1 \mathrm{H}, \mathrm{H}_{5^{\prime}}\right), 4.336\left(\mathrm{dd}, 1 \mathrm{H}, \mathrm{H}_{6}\right)$, 6.225-7.289 (m, 10H, Ar-H) ppm. ${ }^{13} \mathrm{C}$ NMR $\left(\mathrm{CDCl}_{3}-\mathrm{d}_{6}, \mathrm{TMS}\right) \delta$ : $164.34\left(\mathrm{C}_{2}\right), 51.67\left(\mathrm{C}_{4}\right)$, 47.76 $\left(\mathrm{C}_{5}\right), 66.43\left(\mathrm{C}_{6}\right), 124.26-139.39$ (Ar-C)ppm.

13. 4-(4-Bromo-1-naphthyl)-5,6-dihydro-6-(3-chlorophenyl)- ${ }^{4} H$-1,3-oxazine-2-amine: FTIR (KBr): $3548(\mathrm{NH}), 1614(\mathrm{C}=\mathrm{N}), 1215(\mathrm{C}-\mathrm{O}-\mathrm{C}) \mathrm{cm}^{-1} .{ }^{1} \mathrm{H}$ NMR $\left(\mathrm{CDCl}_{3}-\mathrm{d}_{6}, \mathrm{TMS}\right) \delta: 2.215$ (s, 1H, NH $\left.\mathrm{N}_{2}\right), 2.321\left(\mathrm{dd}, 1 \mathrm{H}, \mathrm{H}_{4}\right), 2.421\left(\mathrm{dd}, 1 \mathrm{H}, \mathrm{H}_{5}\right), 2.265$ (dd, 1H, $\left.\mathrm{H}_{5^{\prime}}\right), 4.323\left(\mathrm{dd}, 1 \mathrm{H}, \mathrm{H}_{6}\right)$, 6.232-7.278 (m, 10H, Ar-H) ppm. ${ }^{13} \mathrm{C}$ NMR $\left(\mathrm{CDCl}_{3}-\mathrm{d}_{6}, \mathrm{TMS}\right) \delta$ : $164.33\left(\mathrm{C}_{2}\right), 51.23\left(\mathrm{C}_{4}\right)$, 47.87( $\left.\mathrm{C}_{5}\right), 66.34\left(\mathrm{C}_{6}\right), 126.23-139.15(\mathrm{Ar}-\mathrm{C}) \mathrm{ppm}$.

14. 4-(4-Bromo-1-naphthyl)-5,6-dihydro-6-(4-chlorophenyl)- ${ }^{4} H$-1,3-oxazine-2-amine: FTIR (KBr): $3552(\mathrm{NH}), 1610(\mathrm{C}=\mathrm{N}), 1232(\mathrm{C}-\mathrm{O}-\mathrm{C}) \mathrm{cm}^{-1} .{ }^{1} \mathrm{H}$ NMR $\left(\mathrm{CDCl}_{3}-\mathrm{d}_{6}, \mathrm{TMS}\right) \delta: 2.217$ (s, $\left.1 \mathrm{H}, \mathrm{NH}_{2}\right), 2.323\left(\mathrm{dd}, 1 \mathrm{H}, \mathrm{H}_{4}\right), 2.420\left(\mathrm{dd}, 1 \mathrm{H}, \mathrm{H}_{5}\right), 2.238\left(\mathrm{dd}, 1 \mathrm{H}, \mathrm{H}_{5^{\prime}}\right), 4.398\left(\mathrm{dd}, 1 \mathrm{H}, \mathrm{H}_{6}\right)$, 6.243-7.267 (m, 10H, Ar-H) ppm. ${ }^{13} \mathrm{C}$ NMR $\left(\mathrm{CDCl}_{3}-\mathrm{d}_{6}, \mathrm{TMS}\right) \delta$ : $164.34\left(\mathrm{C}_{2}\right), 51.27\left(\mathrm{C}_{4}\right)$, 47.76( $\left.\mathrm{C}_{5}\right), 66.98\left(\mathrm{C}_{6}\right), 126.37-139.87(\mathrm{Ar}-\mathrm{C}) \mathrm{ppm}$.

15. 4-(4-Chloro-1-naphthyl)-5,6-dihydro-6-(3-bromophenyl) $-{ }^{4} H$-1,3-oxazine-2-amine: FTIR (KBr): 3552(NH), 1623(C=N), $1209(\mathrm{C}-\mathrm{O}-\mathrm{C}) \mathrm{cm}^{-1} .{ }^{1} \mathrm{H}$ NMR $\left(\mathrm{CDCl}_{3}-\mathrm{d}_{6}, \mathrm{TMS}\right) \delta: 2.221$ (s, $\left.\left.1 \mathrm{H}, \mathrm{NH}_{2}\right), 2.314\left(\mathrm{dd}, 1 \mathrm{H}, \mathrm{H}_{4}\right), 2.22 \mathrm{dd}, 1 \mathrm{H}, \mathrm{H}_{5}\right), 2.236\left(\mathrm{dd}, 1 \mathrm{H}, \mathrm{H}_{5^{\prime}}\right), 4.336\left(\mathrm{dd}, 1 \mathrm{H}, \mathrm{H}_{6}\right)$, 6.225-7.289 (m, 10H, Ar-H) ppm. ${ }^{13} \mathrm{C}$ NMR $\left(\mathrm{CDCl}_{3}-\mathrm{d}_{6}, \mathrm{TMS}\right) \delta$ : $164.34\left(\mathrm{C}_{2}\right), 51.67\left(\mathrm{C}_{4}\right)$, 47.76( $\left.\mathrm{C}_{5}\right), 66.43\left(\mathrm{C}_{6}\right), 124.26-139.39$ (Ar-C)ppm. 
16. 4-(4-Chloro-1-naphthyl)-5,6-dihydro-6-(3-chlorophenyl)- ${ }^{4} H$-1,3-oxazine-2-amine: FTIR (KBr): $3548(\mathrm{NH}), 1614(\mathrm{C}=\mathrm{N}), 1215(\mathrm{C}-\mathrm{O}-\mathrm{C}) \mathrm{cm}^{-1} .{ }^{1} \mathrm{H}$ NMR $\left(\mathrm{CDCl}_{3}-\mathrm{d}_{6}, \mathrm{TMS}\right) \delta: 2.215$ (s, $\left.1 \mathrm{H}, \mathrm{NH}_{2}\right), 2.321\left(\mathrm{dd}, 1 \mathrm{H}, \mathrm{H}_{4}\right), 2.421\left(\mathrm{dd}, 1 \mathrm{H}, \mathrm{H}_{5}\right), 2.265\left(\mathrm{dd}, 1 \mathrm{H}, \mathrm{H}_{5^{\prime}}\right), 4.323\left(\mathrm{dd}, 1 \mathrm{H}, \mathrm{H}_{6}\right)$, 6.232-7.278 (m, 10H, Ar-H) ppm. ${ }^{13} \mathrm{C} \mathrm{NMR}\left(\mathrm{CDCl}_{3}-\mathrm{d}_{6}, \mathrm{TMS}\right) \delta$ : $164.33\left(\mathrm{C}_{2}\right), 51.23\left(\mathrm{C}_{4}\right)$, 47.87( $\left.\mathrm{C}_{5}\right), 66.34\left(\mathrm{C}_{6}\right), 126.23-139.15(\mathrm{Ar}-\mathrm{C}) \mathrm{ppm}$.

17. 4-(4-Chloro-1-naphthyl)-5,6-dihydro-6-(4-chlorophenyl)- ${ }^{4} H$-1,3-oxazine-2-amine: FTIR (KBr): 3552(NH), 1610(C=N), 1232(C-O-C) $\mathrm{cm}^{-1} .{ }^{1} \mathrm{H}$ NMR $\left(\mathrm{CDCl}_{3}-\mathrm{d}_{6}, \mathrm{TMS}\right) \delta: 2.217$ (s, 1H, NH $\left.\mathrm{N}_{2}\right), 2.323\left(\mathrm{dd}, 1 \mathrm{H}, \mathrm{H}_{4}\right), 2.420\left(\mathrm{dd}, 1 \mathrm{H}, \mathrm{H}_{5}\right), 2.238\left(\mathrm{dd}, 1 \mathrm{H}, \mathrm{H}_{5^{\prime}}\right), 4.398\left(\mathrm{dd}, 1 \mathrm{H}, \mathrm{H}_{6}\right)$, 6.243-7.267 (m, 10H, Ar-H) ppm. ${ }^{13} \mathrm{C}$ NMR $\left(\mathrm{CDCl}_{3}-\mathrm{d}_{6}, \mathrm{TMS}\right) \delta$ : $164.34\left(\mathrm{C}_{2}\right), 51.27\left(\mathrm{C}_{4}\right)$, 47.76( $\left.\mathrm{C}_{5}\right), 66.98\left(\mathrm{C}_{6}\right), 126.37-139.87(\mathrm{Ar}-\mathrm{C}) \mathrm{ppm}$.

18. 4-(4-Methoxy-1-naphthyl)-5,6-dihydro-6-(3-bromophenyl) $-{ }^{4} H$-1,3-oxazine-2-amine: FTIR (KBr): $3545(\mathrm{NH}), 1605(\mathrm{C}=\mathrm{N}), 1215(\mathrm{C}-\mathrm{O}-\mathrm{C}) \mathrm{cm}^{-1} .{ }^{1} \mathrm{H}$ NMR $\left(\mathrm{CDCl}_{3}-\mathrm{d}_{6}, \mathrm{TMS}\right) \delta: 2.225$ $\left(\mathrm{s}, 1 \mathrm{H}, \mathrm{NH}_{2}\right), 2.219\left(\mathrm{dd}, 1 \mathrm{H}, \mathrm{H}_{4}\right), 2.265\left(\mathrm{dd}, 1 \mathrm{H}, \mathrm{H}_{5}\right), 2.025\left(\mathrm{dd}, 1 \mathrm{H}, \mathrm{H}_{5^{\prime}}\right), 4.632\left(\mathrm{dd}, 1 \mathrm{H}, \mathrm{H}_{6}\right)$, 3.657(s, 3H, $\left.\mathrm{OCH}_{3}\right), 6.725-7.787(\mathrm{~m}, 10 \mathrm{H}, \mathrm{Ar}-\mathrm{H}) \mathrm{ppm} .{ }^{13} \mathrm{C} \mathrm{NMR}\left(\mathrm{CDCl}_{3}-\mathrm{d}_{6}, \mathrm{TMS}\right) \delta: 164.25$ $\left(\mathrm{C}_{2}\right), 51.65\left(\mathrm{C}_{4}\right), 47.20\left(\mathrm{C}_{5}\right), 67.66\left(\mathrm{C}_{6}\right),\left(66.98, \mathrm{OCH}_{3}\right), 121.24-141.39$ (Ar-C)ppm.

19. 4-(4-Methoxy-1-naphthyl)-5,6-dihydro-6-(3-chlorophenyl) $-{ }^{4} H$-1,3-oxazine-2-amine: FTIR (KBr): $3535(\mathrm{NH}), 1589(\mathrm{C}=\mathrm{N}), 1212(\mathrm{C}-\mathrm{O}-\mathrm{C}) \mathrm{cm}^{-1} .{ }^{1} \mathrm{H}$ NMR $\left(\mathrm{CDCl}_{3}-\mathrm{d}_{6}, \mathrm{TMS}\right) \delta: 2.125$ $\left(\mathrm{s}, 1 \mathrm{H}, \mathrm{NH}_{2}\right), 2.258\left(\mathrm{dd}, 1 \mathrm{H}, \mathrm{H}_{4}\right), 2.264\left(\mathrm{dd}, 1 \mathrm{H}, \mathrm{H}_{5}\right), 2.038\left(\mathrm{dd}, 1 \mathrm{H}, \mathrm{H}_{5^{\prime}}\right), 4.671\left(\mathrm{dd}, 1 \mathrm{H}, \mathrm{H}_{6}\right)$, $3.577\left(\mathrm{~s}, 3 \mathrm{H}, \mathrm{CH}_{3}\right), 6.825-7.887(\mathrm{~m}, 10 \mathrm{H}, \mathrm{Ar}-\mathrm{H}) \mathrm{ppm} .{ }^{13} \mathrm{C} \mathrm{NMR}\left(\mathrm{CDCl}_{3}-\mathrm{d}_{6}, \mathrm{TMS}\right) \delta$ : 165.28( $\left.\mathrm{C}_{2}\right), 52.87\left(\mathrm{C}_{4}\right), 47.35\left(\mathrm{C}_{5}\right), 66.89\left(\mathrm{C}_{6}\right),\left(67.98, \mathrm{OCH}_{3}\right), 118.46-139.67$ (Ar-C)ppm.

20. 4-(4-Methoxy-1-naphthyl)-5,6-dihydro-6-(4-chlorophenyl) $-{ }^{4} H$-1,3-oxazine-2-amine: FTIR (KBr): 3555(NH), 1608(C=N), $1215(\mathrm{C}-\mathrm{O}-\mathrm{C}) \mathrm{cm}^{-1} .{ }^{1} \mathrm{H}$ NMR $\left(\mathrm{CDCl}_{3}-\mathrm{d}_{6}, \mathrm{TMS}\right) \delta: 2.112$ $\left(\mathrm{s}, 1 \mathrm{H}, \mathrm{NH}_{2}\right), 2.232\left(\mathrm{dd}, 1 \mathrm{H}, \mathrm{H}_{4}\right), 2.322\left(\mathrm{dd}, 1 \mathrm{H}, \mathrm{H}_{5}\right), 2.154\left(\mathrm{dd}, 1 \mathrm{H}, \mathrm{H}_{5^{\prime}}\right), 4.776\left(\mathrm{dd}, 1 \mathrm{H}, \mathrm{H}_{6}\right)$, 3.087(s, 3H, $\left.\mathrm{CH}_{3}\right), 6.667-7.946(\mathrm{~m}, 10 \mathrm{H}, \mathrm{Ar}-\mathrm{H}) \mathrm{ppm} .{ }^{13} \mathrm{C} \mathrm{NMR}\left(\mathrm{CDCl}_{3}-\mathrm{d}_{6}, \mathrm{TMS}\right) \delta$ : 164.87( $\left(\mathrm{C}_{2}\right), 52.32\left(\mathrm{C}_{4}\right), 47.443\left(\mathrm{C}_{5}\right), 66.56\left(\mathrm{C}_{6}\right),\left(65.98, \mathrm{OCH}_{3}\right), 118.57-139.79(\mathrm{Ar}-\mathrm{C}) \mathrm{ppm}$.

21. 4-(4-Methyl-1-naphthyl)-5,6-dihydro-6-(3-bromophenyl) $\quad{ }_{-}^{4} H$-1,3-oxazine-2-amine: FTIR (KBr): 3543(NH), 1597(C=N), 1213(C-O-C) $\mathrm{cm}^{-1} .{ }^{1} \mathrm{H}$ NMR $\left(\mathrm{CDCl}_{3}-\mathrm{d}_{6}, \mathrm{TMS}\right) \delta: 2.219$ $\left(\mathrm{s}, 1 \mathrm{H}, \mathrm{NH}_{2}\right), 2.207\left(\mathrm{dd}, 1 \mathrm{H}, \mathrm{H}_{4}\right), 2.257\left(\mathrm{dd}, 1 \mathrm{H}, \mathrm{H}_{5}\right), 2.019\left(\mathrm{dd}, 1 \mathrm{H}, \mathrm{H}_{5^{\prime}}\right), 4.646\left(\mathrm{dd}, 1 \mathrm{H}, \mathrm{H}_{6}\right)$, 4.687(s, 3H, $\left.\mathrm{CH}_{3}\right), 6.657-7.757(\mathrm{~m}, 10 \mathrm{H}, \mathrm{Ar}-\mathrm{H}) \mathrm{ppm} .{ }^{13} \mathrm{C} \mathrm{NMR}\left(\mathrm{CDCl}_{3}-\mathrm{d}_{6}, \mathrm{TMS}\right) \delta: 164.18$ $\left(\mathrm{C}_{2}\right), 51.43\left(\mathrm{C}_{4}\right), 47.45\left(\mathrm{C}_{5}\right), 67.67\left(\mathrm{C}_{6}\right), 25.98\left(\mathrm{CH}_{3}\right), 121.24-141.39$ (Ar-C)ppm.

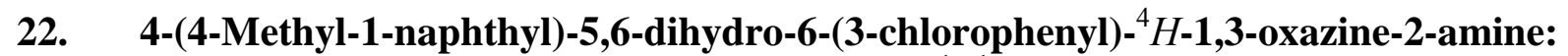
FTIR (KBr): $3538(\mathrm{NH}), 1597(\mathrm{C}=\mathrm{N}), 1233(\mathrm{C}-\mathrm{O}-\mathrm{C}) \mathrm{cm}^{-1} .{ }^{1} \mathrm{H}$ NMR $\left(\mathrm{CDCl}_{3}-\mathrm{d}_{6}, \mathrm{TMS}\right) \delta: 2.122$ $\left(\mathrm{s}, 1 \mathrm{H}, \mathrm{NH}_{2}\right), 2.256\left(\mathrm{dd}, 1 \mathrm{H}, \mathrm{H}_{4}\right), 2.265\left(\mathrm{dd}, 1 \mathrm{H}, \mathrm{H}_{5}\right), 2.044\left(\mathrm{dd}, 1 \mathrm{H}, \mathrm{H}_{5^{\prime}}\right), 4.664\left(\mathrm{dd}, 1 \mathrm{H}, \mathrm{H}_{6}\right)$, 4.534(s, 3H, CH $\left.\mathrm{CH}_{3}\right), 6.823-7.877(\mathrm{~m}, 10 \mathrm{H}, \mathrm{Ar}-\mathrm{H}) \mathrm{ppm} .{ }^{13} \mathrm{C} \mathrm{NMR}\left(\mathrm{CDCl}_{3}-\mathrm{d}_{6}, \mathrm{TMS}\right) \delta$ : 165.68( $\left(\mathrm{C}_{2}\right), 52.887\left(\mathrm{C}_{4}\right), 47.55\left(\mathrm{C}_{5}\right), 66.86\left(\mathrm{C}_{6}\right), 26.48\left(\mathrm{CH}_{3}\right), 118.67-139.48(\mathrm{Ar}-\mathrm{C}) \mathrm{ppm}$.

23. 4-(4-Methyl-1-naphthyl)-5,6-dihydro-6-(4-chlorophenyl) $-{ }^{4} H$-1,3-oxazine-2-amine: FTIR (KBr): 3556(NH), 1618(C=N), $1217(\mathrm{C}-\mathrm{O}-\mathrm{C}) \mathrm{cm}^{-1} .{ }^{1} \mathrm{H}$ NMR $\left(\mathrm{CDCl}_{3}-\mathrm{d}_{6}, \mathrm{TMS}\right) \delta: 2.121$ $\left(\mathrm{s}, 1 \mathrm{H}, \mathrm{NH}_{2}\right), 2.235\left(\mathrm{dd}, 1 \mathrm{H}, \mathrm{H}_{4}\right), 2.329\left(\mathrm{dd}, 1 \mathrm{H}, \mathrm{H}_{5}\right), 2.134\left(\mathrm{dd}, 1 \mathrm{H}, \mathrm{H}_{5^{\prime}}\right), 4.775\left(\mathrm{dd}, 1 \mathrm{H}, \mathrm{H}_{6}\right)$, 4.034(s, 3H, $\left.\mathrm{CH}_{3}\right), 6.634-7.936(\mathrm{~m}, 10 \mathrm{H}, \mathrm{Ar}-\mathrm{H}) \mathrm{ppm} .{ }^{13} \mathrm{C} \mathrm{NMR}\left(\mathrm{CDCl}_{3}-\mathrm{d}_{6}, \mathrm{TMS}\right) \delta$ : 164.56 $\left(\mathrm{C}_{2}\right), 52.67\left(\mathrm{C}_{4}\right), 47.420\left(\mathrm{C}_{5}\right), 66.57\left(\mathrm{C}_{6}\right), 25.92\left(\mathrm{CH}_{3}\right), 118.59-139.92(\mathrm{Ar}-\mathrm{C}) \mathrm{ppm}$.

24. 4-(2-Naphthyl)-5,6-dihydro-6-(3-bromophenyl)- ${ }^{4} H$-1,3-oxazine-2-amine: FTIR (KBr): 3548(NH), 1603(C=N), 1218(C-O-C) $\mathrm{cm}^{-1} .{ }^{1} \mathrm{H}$ NMR $\left(\mathrm{CDCl}_{3}-\mathrm{d}_{6}, \mathrm{TMS}\right) \delta: 2.225\left(\mathrm{~s}, 1 \mathrm{H}, \mathrm{NH}_{2}\right)$, $2.219\left(\mathrm{dd}, 1 \mathrm{H}, \mathrm{H}_{4}\right), 2.262\left(\mathrm{dd}, 1 \mathrm{H}, \mathrm{H}_{5}\right), 2.034\left(\mathrm{dd}, 1 \mathrm{H}, \mathrm{H}_{5^{\prime}}\right), 4.690\left(\mathrm{dd}, 1 \mathrm{H}, \mathrm{H}_{6}\right), 6.645-7.738(\mathrm{~m}$, 


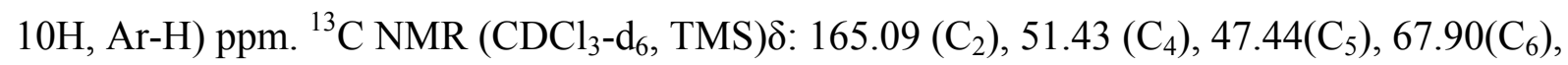
25.36( $\left(\mathrm{CH}_{3}\right), 121.36-141.48(\mathrm{Ar}-\mathrm{C}) \mathrm{ppm}$.

25. 4-(2-Naphthyl)-5,6-dihydro-6-(3-chlorophenyl)- ${ }^{4} H$-1,3-oxazine-2-amine: FTIR (KBr): $3545(\mathrm{NH}), 1605(\mathrm{C}=\mathrm{N}), 1215(\mathrm{C}-\mathrm{O}-\mathrm{C}) \mathrm{cm}^{-1} .{ }^{1} \mathrm{H} \mathrm{NMR}\left(\mathrm{CDCl}_{3}-\mathrm{d}_{6}, \mathrm{TMS}\right) \delta: 2.225\left(\mathrm{~s}, 1 \mathrm{H}, \mathrm{NH}_{2}\right)$, $2.219\left(\mathrm{dd}, 1 \mathrm{H}, \mathrm{H}_{4}\right), 2.265\left(\mathrm{dd}, 1 \mathrm{H}, \mathrm{H}_{5}\right), 2.025\left(\mathrm{dd}, 1 \mathrm{H}, \mathrm{H}_{5^{\prime}}\right), 4.632\left(\mathrm{dd}, 1 \mathrm{H}, \mathrm{H}_{6}\right), 6.725-7.788(\mathrm{~m}$, $10 \mathrm{H}, \mathrm{Ar}-\mathrm{H}) \mathrm{ppm} .{ }^{13} \mathrm{C} \mathrm{NMR}\left(\mathrm{CDCl}_{3}-\mathrm{d}_{6}, \mathrm{TMS}\right) \delta$ : $165.25\left(\mathrm{C}_{2}\right), 52.65\left(\mathrm{C}_{4}\right), 47.16\left(\mathrm{C}_{5}\right), 67.32\left(\mathrm{C}_{6}\right)$, 121.85-138.32 (Ar-C)ppm.

26. 4-(2-Naphthyl)-5,6-dihydro-6-(3-chlorophenyl)- ${ }^{4} H$-1,3-oxazine-2-amine: FTIR (KBr): $3535(\mathrm{NH}), 1589(\mathrm{C}=\mathrm{N}), 1222(\mathrm{C}-\mathrm{O}-\mathrm{C}) \mathrm{cm}^{-1} .{ }^{1} \mathrm{H} \mathrm{NMR}\left(\mathrm{CDCl}_{3}-\mathrm{d}_{6}, \mathrm{TMS}\right) \delta: 2.125\left(\mathrm{~s}, 1 \mathrm{H}, \mathrm{NH}_{2}\right)$, $2.258\left(\mathrm{dd}, 1 \mathrm{H}, \mathrm{H}_{4}\right), 2.264\left(\mathrm{dd}, 1 \mathrm{H}, \mathrm{H}_{5}\right), 2.038\left(\mathrm{dd}, 1 \mathrm{H}, \mathrm{H}_{5^{\prime}}\right), 4.671\left(\mathrm{dd}, 1 \mathrm{H}, \mathrm{H}_{6}\right), 6.825-7.887(\mathrm{~m}$, $10 \mathrm{H}, \mathrm{Ar}-\mathrm{H}) \mathrm{ppm} .{ }^{13} \mathrm{C} \mathrm{NMR}\left(\mathrm{CDCl}_{3}-\mathrm{d}_{6}, \mathrm{TMS}\right) \delta: 165.28\left(\mathrm{C}_{2}\right), 52.87\left(\mathrm{C}_{4}\right), 47.21\left(\mathrm{C}_{5}\right), 67.29\left(\mathrm{C}_{6}\right)$, 121.90-139.38(Ar-C)ppm.

27. 4-(6-Methoxy-2-naphthyl)-5,6-dihydro-6-(3-bromophenyl) ${ }^{4} H$-1,3-oxazine-2-amine: FTIR (KBr): $3537(\mathrm{NH}), 1617(\mathrm{C}=\mathrm{N}), 1223(\mathrm{C}-\mathrm{O}-\mathrm{C}) \mathrm{cm}^{-1} .{ }^{1} \mathrm{H}$ NMR $\left(\mathrm{CDCl}_{3}-\mathrm{d}_{6}, \mathrm{TMS}\right) \delta: 2.217$ $\left(\mathrm{s}, 1 \mathrm{H}, \mathrm{NH}_{2}\right), 2.223\left(\mathrm{dd}, 1 \mathrm{H}, \mathrm{H}_{4}\right), 2.234\left(\mathrm{dd}, 1 \mathrm{H}, \mathrm{H}_{5}\right), 2.024\left(\mathrm{dd}, 1 \mathrm{H}, \mathrm{H}_{5^{\prime}}\right), 4.634\left(\mathrm{dd}, 1 \mathrm{H}, \mathrm{H}_{6}\right)$, 3.656(s, 3H, $\left.\mathrm{OCH}_{3}\right), 6.711-7.723(\mathrm{~m}, 10 \mathrm{H}, \mathrm{Ar}-\mathrm{H}) \mathrm{ppm} .{ }^{13} \mathrm{C} \mathrm{NMR}\left(\mathrm{CDCl}_{3}-\mathrm{d}_{6}, \mathrm{TMS}\right) \delta: 164.56$ $\left(\mathrm{C}_{2}\right), 51.75\left(\mathrm{C}_{4}\right), 47.27\left(\mathrm{C}_{5}\right), 67.86\left(\mathrm{C}_{6}\right),\left(66.43, \mathrm{OCH}_{3}\right), 121.26-141.87$ (Ar-C)ppm.

28. 4-(6-Methoxy-1-naphthyl)-5,6-dihydro-6-(3-chlorophenyl) $-{ }^{4} H$-1,3-oxazine-2-amine: FTIR (KBr): 3533(NH), 1592(C=N), $1218(\mathrm{C}-\mathrm{O}-\mathrm{C}) \mathrm{cm}^{-1} .{ }^{1} \mathrm{H}$ NMR $\left(\mathrm{CDCl}_{3}-\mathrm{d}_{6}, \mathrm{TMS}\right) \delta: 2.123$ (s, $\left.1 \mathrm{H}, \mathrm{NH}_{2}\right), 2.257\left(\mathrm{dd}, 1 \mathrm{H}, \mathrm{H}_{4}\right), 2.278\left(\mathrm{dd}, 1 \mathrm{H}, \mathrm{H}_{5}\right), 2.029\left(\mathrm{dd}, 1 \mathrm{H}, \mathrm{H}_{5^{\prime}}\right), 4.646\left(\mathrm{dd}, 1 \mathrm{H}, \mathrm{H}_{6}\right)$, 3.578(s, 3H, $\left.\mathrm{OCH}_{3}\right), 6.833-7.867(\mathrm{~m}, 10 \mathrm{H}, \mathrm{Ar}-\mathrm{H}) \mathrm{ppm} .{ }^{13} \mathrm{C} \mathrm{NMR}\left(\mathrm{CDCl}_{3}-\mathrm{d}_{6}, \mathrm{TMS}\right) \delta$ : 165.22( $\left(\mathrm{C}_{2}\right), 52.84\left(\mathrm{C}_{4}\right), 47.36\left(\mathrm{C}_{5}\right), 66.80\left(\mathrm{C}_{6}\right),\left(67.66, \mathrm{OCH}_{3}\right), 118.32-139.60(\mathrm{Ar}-\mathrm{C}) \mathrm{ppm}$.

29. 4-(6-Methoxy-1-naphthyl)-5,6-dihydro-6-(4-chlorophenyl)- ${ }^{4} H$-1,3-oxazine-2-amine: FTIR (KBr): 3552(NH), 1623(C=N), $1246(\mathrm{C}-\mathrm{O}-\mathrm{C}) \mathrm{cm}^{-1} .{ }^{1} \mathrm{H}$ NMR $\left(\mathrm{CDCl}_{3}-\mathrm{d}_{6}, \mathrm{TMS}\right) \delta: 2.120$ $\left(\mathrm{s}, 1 \mathrm{H}, \mathrm{NH}_{2}\right), 2.226\left(\mathrm{dd}, 1 \mathrm{H}, \mathrm{H}_{4}\right), 2.334\left(\mathrm{dd}, 1 \mathrm{H}, \mathrm{H}_{5}\right), 2.165\left(\mathrm{dd}, 1 \mathrm{H}, \mathrm{H}_{5^{\prime}}\right), 4.778\left(\mathrm{dd}, 1 \mathrm{H}, \mathrm{H}_{6}\right)$, 3.136(s, 3H, $\left.\mathrm{OCH}_{3}\right), 6.636-7.989(\mathrm{~m}, 10 \mathrm{H}, \mathrm{Ar}-\mathrm{H}) \mathrm{ppm} .{ }^{13} \mathrm{C} \mathrm{NMR}\left(\mathrm{CDCl}_{3}-\mathrm{d}_{6}, \mathrm{TMS}\right) \delta$ : 164.36 $\left(\mathrm{C}_{2}\right), 52.89\left(\mathrm{C}_{4}\right), 47.490\left(\mathrm{C}_{5}\right), 66.80\left(\mathrm{C}_{6}\right),\left(65.40, \mathrm{OCH}_{3}\right), 118.22-139.69$ (Ar-C)ppm.

30. 4-(6-Methyl-1-naphthyl)-5,6-dihydro-6-(3-bromophenyl) $-{ }^{4} H$-1,3-oxazine-2-amine: FTIR (KBr): $3545(\mathrm{NH}), 1583(\mathrm{C}=\mathrm{N}), 1223(\mathrm{C}-\mathrm{O}-\mathrm{C}) \mathrm{cm}^{-1} .{ }^{1} \mathrm{H}$ NMR $\left(\mathrm{CDCl}_{3}-\mathrm{d}_{6}, \mathrm{TMS}\right) \delta: 2.223$ $\left(\mathrm{s}, 1 \mathrm{H}, \mathrm{NH}_{2}\right), 2.212\left(\mathrm{dd}, 1 \mathrm{H}, \mathrm{H}_{4}\right), 2.246\left(\mathrm{dd}, 1 \mathrm{H}, \mathrm{H}_{5}\right), 2.023\left(\mathrm{dd}, 1 \mathrm{H}, \mathrm{H}_{5^{\prime}}\right), 4.667\left(\mathrm{dd}, 1 \mathrm{H}, \mathrm{H}_{6}\right)$,

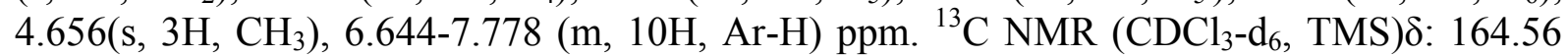
$\left(\mathrm{C}_{2}\right), 51.89\left(\mathrm{C}_{4}\right), 47.45\left(\mathrm{C}_{5}\right), 67.89\left(\mathrm{C}_{6}\right), 25.67\left(\mathrm{CH}_{3}\right), 121.33-141.56(\mathrm{Ar}-\mathrm{C}) \mathrm{ppm}$.

31. 4-(6-Methyl-1-naphthyl)-5,6-dihydro-6-(3-chlorophenyl) $-{ }^{4} H$-1,3-oxazine-2-amine: FTIR (KBr): $3534(\mathrm{NH}), 1593(\mathrm{C}=\mathrm{N}), 1213(\mathrm{C}-\mathrm{O}-\mathrm{C}) \mathrm{cm}^{-1} .{ }^{1} \mathrm{H}$ NMR $\left(\mathrm{CDCl}_{3}-\mathrm{d}_{6}, \mathrm{TMS}\right) \delta: 2.123$ $\left(\mathrm{s}, 1 \mathrm{H}, \mathrm{NH}_{2}\right), 2.246\left(\mathrm{dd}, 1 \mathrm{H}, \mathrm{H}_{4}\right), 2.236\left(\mathrm{dd}, 1 \mathrm{H}, \mathrm{H}_{5}\right), 2.034\left(\mathrm{dd}, 1 \mathrm{H}, \mathrm{H}_{5^{\prime}}\right), 4.665\left(\mathrm{dd}, 1 \mathrm{H}, \mathrm{H}_{6}\right)$, $4.567\left(\mathrm{~s}, 3 \mathrm{H}, \mathrm{CH}_{3}\right), 6.877-7.827(\mathrm{~m}, 10 \mathrm{H}, \mathrm{Ar}-\mathrm{H}) \mathrm{ppm} .{ }^{13} \mathrm{C} \mathrm{NMR}\left(\mathrm{CDCl}_{3}-\mathrm{d}_{6}, \mathrm{TMS}\right) \delta$ : 165.89 $\left(\mathrm{C}_{2}\right), 52.865\left(\mathrm{C}_{4}\right), 47.80\left(\mathrm{C}_{5}\right), 66.76\left(\mathrm{C}_{6}\right), 26.99\left(\mathrm{CH}_{3}\right), 118.15-139.89(\mathrm{Ar}-\mathrm{C}) \mathrm{ppm}$.

32. 4-(6-Methyl-1-naphthyl)-5,6-dihydro-6-(4-chlorophenyl) $-{ }^{4} H$-1,3-oxazine-2-amine: FTIR (KBr): $3552(\mathrm{NH}), 1612(\mathrm{C}=\mathrm{N}), 1213(\mathrm{C}-\mathrm{O}-\mathrm{C}) \mathrm{cm}^{-1} .{ }^{1} \mathrm{H}$ NMR $\left(\mathrm{CDCl}_{3}-\mathrm{d}_{6}, \mathrm{TMS}\right) \delta: 2.115$ $\left(\mathrm{s}, 1 \mathrm{H}, \mathrm{NH}_{2}\right), 2.234\left(\mathrm{dd}, 1 \mathrm{H}, \mathrm{H}_{4}\right), 2.336\left(\mathrm{dd}, 1 \mathrm{H}, \mathrm{H}_{5}\right), 2.136\left(\mathrm{dd}, 1 \mathrm{H}, \mathrm{H}_{5^{\prime}}\right), 4.767\left(\mathrm{dd}, 1 \mathrm{H}, \mathrm{H}_{6}\right)$, 4.032(s, 3H, $\left.\mathrm{OCH}_{3}\right), 6.636-7.947(\mathrm{~m}, 10 \mathrm{H}, \mathrm{Ar}-\mathrm{H}) \mathrm{ppm} .{ }^{13} \mathrm{C} \mathrm{NMR}\left(\mathrm{CDCl}_{3}-\mathrm{d}_{6}, \mathrm{TMS}\right) \delta$ : 164.78( $\left.\mathrm{C}_{2}\right), 52.89\left(\mathrm{C}_{4}\right), 47.490\left(\mathrm{C}_{5}\right), 66.35\left(\mathrm{C}_{6}\right), 25.67\left(\mathrm{CH}_{3}\right)$, 118.89-139.27(Ar-C)ppm. 
33. 4-(1-Pyrenyl)-5,6-dihydro-6-(3-bromophenyl)- ${ }^{4} H$-1,3-oxazine-2-amine: $\mathrm{FTIR}(\mathrm{KBr})$ : $3548(\mathrm{NH}), 1598(\mathrm{C}=\mathrm{N}), 1223(\mathrm{C}-\mathrm{O}-\mathrm{C}) \mathrm{cm}^{-1} .{ }^{1} \mathrm{H}$ NMR $\left(\mathrm{CDCl}_{3}-\mathrm{d}_{6}, \mathrm{TMS}\right) \delta: 2.217\left(\mathrm{~s}, 1 \mathrm{H}, \mathrm{NH}_{2}\right)$, $2.234\left(\mathrm{dd}, 1 \mathrm{H}, \mathrm{H}_{4}\right), 2.267\left(\mathrm{dd}, 1 \mathrm{H}, \mathrm{H}_{5}\right), 2.022\left(\mathrm{dd}, 1 \mathrm{H}, \mathrm{H}_{5^{\prime}}\right), 4.678\left(\mathrm{dd}, 1 \mathrm{H}, \mathrm{H}_{6}\right), 6.637-7.789(\mathrm{~m}$, $10 \mathrm{H}, \mathrm{Ar}-\mathrm{H}) \mathrm{ppm} .{ }^{13} \mathrm{C} \mathrm{NMR}\left(\mathrm{CDCl}_{3}-\mathrm{d}_{6}, \mathrm{TMS}\right) \delta: 164.34\left(\mathrm{C}_{2}\right), 51.78\left(\mathrm{C}_{4}\right), 47.43\left(\mathrm{C}_{5}\right), 67.36$ $\left(\mathrm{C}_{6}\right), 121.57-141.90$ (Ar-C)ppm.

34. 4-(1-Pyrenyl)-5,6-dihydro-6-(3-chlorophenyl)- ${ }^{4} H$-1,3-oxazine-2-amine:FTIR(KBr): 3546(NH), 1594(C=N), 1245(C-O-C) cm ${ }^{-1} .{ }^{1} \mathrm{H}$ NMR $\left(\mathrm{CDCl}_{3}-\mathrm{d}_{6}, \mathrm{TMS}\right) \delta: 2.118\left(\mathrm{~s}, 1 \mathrm{H}, \mathrm{NH}_{2}\right)$, 2.335 (dd, $\left.1 \mathrm{H}, \mathrm{H}_{4}\right), 2.232\left(\mathrm{dd}, 1 \mathrm{H}, \mathrm{H}_{5}\right), 2.045\left(\mathrm{dd}, 1 \mathrm{H}, \mathrm{H}_{5^{\prime}}\right), 4.667\left(\mathrm{dd}, 1 \mathrm{H}, \mathrm{H}_{6}\right), 6.832-7.810(\mathrm{~m}$, $10 \mathrm{H}, \mathrm{Ar}-\mathrm{H}) \mathrm{ppm} .{ }^{13} \mathrm{C} \mathrm{NMR}\left(\mathrm{CDCl}_{3}-\mathrm{d}_{6}, \mathrm{TMS}\right) \delta: 165.12\left(\mathrm{C}_{2}\right), 52.848\left(\mathrm{C}_{4}\right), 47.32\left(\mathrm{C}_{5}\right), 66.76$ $\left(\mathrm{C}_{6}\right), 118.12-139.67$ (Ar-C)ppm.

35. 4-(4-Methyl-1-naphthyl)-5,6-dihydro-6-(4-chlorophenyl) $-{ }^{4} H$-1,3-oxazine-2-amine: FTIR(KBr): 3532(NH), 1623(C=N), $1256(\mathrm{C}-\mathrm{O}-\mathrm{C}) \mathrm{cm}^{-1} .{ }^{1} \mathrm{H}$ NMR $\left(\mathrm{CDCl}_{3}-\mathrm{d}_{6}, \mathrm{TMS}\right) \delta: 2.122$ $\left(\mathrm{s}, 1 \mathrm{H}, \mathrm{NH}_{2}\right), 2.234\left(\mathrm{dd}, 1 \mathrm{H}, \mathrm{H}_{4}\right), 2.343\left(\mathrm{dd}, 1 \mathrm{H}, \mathrm{H}_{5}\right), 2.139\left(\mathrm{dd}, 1 \mathrm{H}, \mathrm{H}_{5^{\prime}}\right), 4.736\left(\mathrm{dd}, 1 \mathrm{H}, \mathrm{H}_{6}\right)$, 6.634-7.909(m, 10H, Ar-H) ppm. ${ }^{13} \mathrm{C} \mathrm{NMR}\left(\mathrm{CDCl}_{3}-\mathrm{d}_{6}, \mathrm{TMS}\right) \delta: 164.34\left(\mathrm{C}_{2}\right), 52.56\left(\mathrm{C}_{4}\right)$, 47.480( $\left.\mathrm{C}_{5}\right), 66.48\left(\mathrm{C}_{6}\right), 118.34-139.78(\mathrm{ArC}) \mathrm{ppm}$.

\section{3. Measurement of Insect antifeedant activities}

This test was performed with a $4^{\text {th }}$ instar larva Achoea janata L against castor semilooper, were reared as described on the leaves of castor, Ricinus communis in the laboratory at the temperature range of $26{ }^{\circ} \mathrm{C} \pm 1{ }^{\circ} \mathrm{C}$ and a relative humidity of $75-85 \%$. The leaf - disc bioassay method [28] was used against the $4^{\text {th }}$ instar larvae to measure the antifeedant activity. The $4^{\text {th }}$ instar larvae were selected for testing because the larvae at this stage feed very voraciously. Castor leaf discs of a diameter of $1.85 \mathrm{~cm}$ were punched and intact with the petioles. All synthesized oxazine-2-amines were dissolved in acetone at a concentration of $200 \mathrm{ppm}$ dipped for 5 minutes. The leaf discs were air-dried and placed in one litre beaker containing little water in order to facilitate translocation of water. Therefore, the leaf discs remain fresh throughout the duration of the rest, $4^{\text {th }}$ instar larvae of the test insect, which had been preserved on the leaf discs of all oxazine-2-amines and allowed to feed on them for $24 \mathrm{~h}$. The areas of the leaf disc consumed were measured by Dethler's method [22]. The observed oxazine-2-amines were presented in Table 2

Table 2. The insect antifeedant activities of the 4-(aryl)-5,6-dihydro-6(substituted phenyl) ${ }^{4} H-1,3-$ oxazine-2-amines.

\begin{tabular}{|c|c|c|c|c|c|c|c|c|c|c|}
\hline Entry & $\begin{array}{c}\mathbf{4 - 6} \\
\mathbf{p m}\end{array}$ & $\begin{array}{c}\mathbf{6 - 8} \\
\mathbf{p m}\end{array}$ & $\begin{array}{c}\mathbf{8 - 1 0} \\
\mathbf{p m}\end{array}$ & $\begin{array}{c}\mathbf{1 0 - 2} \\
\mathbf{p m}\end{array}$ & $\begin{array}{c}\mathbf{1 2 - 6} \\
\mathbf{a m}\end{array}$ & $\begin{array}{c}\mathbf{6 - 8} \\
\mathbf{a m}\end{array}$ & $\begin{array}{c}\mathbf{8 a m}- \\
\mathbf{1 2 N n}\end{array}$ & $\begin{array}{c}\mathbf{1 2 N n}- \\
\mathbf{2 p m}\end{array}$ & $\begin{array}{c}\mathbf{2 - 4} \\
\mathbf{p m}\end{array}$ & $\begin{array}{c}\text { Total leaf disc } \\
\text { consumed in 24 h }\end{array}$ \\
\hline $\mathbf{1}$ & 1 & 1 & 1 & 0.5 & 0.5 & 1 & 1 & 1 & 1 & 8 \\
\hline $\mathbf{2}$ & 2 & 10.5 & 1 & 1 & 1 & 1 & 1 & 0 & 0.5 & 9 \\
\hline $\mathbf{3}$ & 1 & 2 & 2 & 1 & 0 & 0 & 1 & 1 & 1 & 9 \\
\hline $\mathbf{4}$ & 0.5 & 1 & 1 & 0.25 & 0.5 & 0.5 & 0.5 & 1 & 1 & 6 \\
\hline $\mathbf{5}$ & 1 & 1 & 1 & 1 & 0.5 & 0.5 & 0.5 & 1 & 0.5 & 7 \\
\hline $\mathbf{6}$ & 1 & 1 & 1 & 1 & 0 & 0.5 & 0.5 & 1 & 0.5 & 6.5 \\
\hline $\mathbf{7}$ & 1 & 0.5 & 1 & 1 & 1 & 1 & 0.5 & 0.5 & 0.5 & 7 \\
\hline $\mathbf{8}$ & 1 & 1 & 1 & 0.25 & 0.25 & 1 & 0.5 & 1 & 0.5 & 7 \\
\hline
\end{tabular}




\begin{tabular}{|c|c|c|c|c|c|c|c|c|c|c|}
\hline 9 & 1 & 0.5 & 1 & 1 & 1 & 0.5 & 1 & 0.5 & 0.5 & 7 \\
\hline 10 & 1 & 1 & 0.5 & 1 & 0.5 & 0.5 & 0.5 & 0.5 & 0.5 & 6 \\
\hline 11 & 1 & 0.5 & 1 & 0.5 & 0.5 & 1 & 1 & 1 & 1 & 8 \\
\hline 12 & 0.5 & 0.25 & 0.5 & 0.25 & 0.5 & 0.5 & 0.5 & 1 & 1 & 5 \\
\hline 13 & 0.25 & 0.25 & 0.25 & 0.25 & 0.5 & 0.5 & 0.5 & 1 & 0.5 & 4 \\
\hline 14 & 0.25 & 0.25 & 0.25 & 0.25 & 0.25 & 0.5 & 0.25 & 1 & 0.5 & 3.5 \\
\hline 15 & 0.25 & 0.25 & 0.25 & 0.25 & 0.5 & 0.5 & 0.5 & 1 & 0.5 & 4 \\
\hline 16 & 0.25 & 0.25 & 0.25 & 0.25 & 0.25 & 0.5 & 0.25 & 1 & 0.5 & 3.5 \\
\hline 17 & 0.25 & 0.25 & 0 & 0.25 & 0.5 & 0 & 0.25 & 1 & 0.5 & 3 \\
\hline 18 & 0.25 & 0.25 & 0.25 & 0.25 & 0.5 & 0.5 & 0.5 & 1 & 0.5 & 4 \\
\hline 19 & 0.25 & 0.25 & 0.25 & 0.25 & 0.25 & 0.5 & 0.25 & 1 & 0.5 & 3.5 \\
\hline 20 & 1 & 0.5 & 1 & 1 & 1 & 1 & 0.5 & 0.5 & 0.5 & 7 \\
\hline 21 & 1 & 1 & 1 & 0.25 & 0.25 & 1 & 0.5 & 1 & 0.5 & 7 \\
\hline 22 & 1 & 0.5 & 1 & 1 & 1 & 0.5 & 1 & 0.5 & 0.5 & 7 \\
\hline 23 & 1 & 1 & 0.5 & 1 & 0.5 & 0.5 & 0.5 & 0.5 & 0.5 & 6 \\
\hline 24 & 1 & 0.5 & 1 & 0.5 & 0.5 & 1 & 1 & 1 & 1 & 8 \\
\hline 15 & 2 & 1 & 1 & 0.5 & 0.5 & 1 & 1 & 1 & 1 & 9 \\
\hline 26 & 1 & 0.5 & 1 & 0.5 & 0.5 & 1 & 1 & 1 & 1 & 8 \\
\hline 27 & 2 & 2 & 1 & 0.5 & 0.5 & 1 & 1 & 1 & 1 & 10 \\
\hline 28 & 2 & 2 & 1 & 0.5 & 1 & 1 & 1 & 1 & 0.5 & 11 \\
\hline 29 & 1 & 2 & 2 & 1 & 0 & 0 & 1 & 1 & 1 & 9 \\
\hline 30 & 1 & 1 & 1 & 1 & 0.5 & 0.5 & 0.5 & 1 & 1 & 8 \\
\hline 31 & 1 & 0.5 & 1 & 1 & 0.5 & 0.5 & 0.5 & 1 & 0.5 & 7.5 \\
\hline 32 & 2 & 1 & 1 & 0.5 & 1 & 0.5 & 0.5 & 1 & 0.5 & 8 \\
\hline 33 & 1 & 0.5 & 1 & 1 & 1 & 1 & 0.5 & 0.5 & 0.5 & 7 \\
\hline 34 & 1 & 1 & 1 & 0.25 & 0.25 & 1 & 0.5 & 1 & 0.5 & 7 \\
\hline 35 & 1 & 0.5 & 1 & 1 & 1 & 0.5 & 1 & 0.5 & 0.5 & 7 \\
\hline
\end{tabular}

\section{RESULTS AND DISCUSSION}

The results of the antifeedant activity of oxazine-2-amines are presented in Table 2 reveals that all halogenated compounds were found to reflect satisfactory insect antifeedant activities. This test was performed with the insects which ate only two-leaf disc soaked under the solution of this compound. All compounds were active for insect antifeedant activities. The oxazine derivatives 12-17 4-(4-chloro-1-naphthyl) and 4(4-bromo-1-naphthyl)-5,6dihydro-6(substituted phenyl)- ${ }^{4} H-1,3$-oxazine-2-amines showed enough antifeedant activity. Further compounds 12-17 was subjected to measure the antifeedant activity at different 50, $100,150 \mathrm{ppm}$ concentrations and the observation reveals that as the concentrations decreased, 
the activity also decreased. It is observed from the results in Table 3 and that the oxazine 1217 showed an appreciable antifeedant activity at $150 \mathrm{ppm}$ concentration.

Table 3. Antifeedant activity of compound 12-17 4-(4-chloro-1-naphthyl) and 4(4-bromo-1naphthyl)-5,6-dihydro-6(substituted phenyl)-4H-1,3-oxazine-2-amines showed an appreciable antifeedant activity at 3 different concentrations.

\begin{tabular}{|c|c|c|c|c|c|c|c|c|c|c|}
\hline Entry & $\begin{array}{c}4-6 \\
\mathrm{pm}\end{array}$ & $\begin{array}{c}6-8 \\
\mathrm{pm}\end{array}$ & $\begin{array}{c}8-10 \\
\mathrm{pm}\end{array}$ & $\begin{array}{c}10-2 \\
\mathrm{pm}\end{array}$ & $\begin{array}{c}12-6 \\
\mathrm{am}\end{array}$ & $\begin{array}{c}6-8 \\
\mathrm{am}\end{array}$ & $\begin{array}{c}8 \mathrm{am}- \\
12 \mathrm{Nn}\end{array}$ & $\begin{array}{c}12 \mathrm{Nn}- \\
2 \mathrm{pm}\end{array}$ & $\begin{array}{c}2-4 \\
\mathrm{pm}\end{array}$ & $\begin{array}{c}\text { Total leaf disc } \\
\text { consumed in 24 h }\end{array}$ \\
\hline $\mathbf{5 0}$ & 0.25 & 0 & 0.25 & 0.25 & 0 & 0 & 0 & 0 & 0 & 0.75 \\
\hline $\mathbf{1 0 0}$ & 0.25 & 0.25 & 0 & 0 & 0 & 0 & 0 & 0 & 0 & 0.5 \\
\hline $\mathbf{1 5 0}$ & 0.25 & 0 & 0 & 0 & 0 & 0 & 0 & 0 & 0 & 0.25 \\
\hline
\end{tabular}

umber of leaf discs consumed by the insect (Values are mean + SE of five).

\section{CONCLUSIONS}

Some unsaturated 1,3-oxazine derivatives have been synthesised and characterized by their physical constants and spectroscopic data. The insect antifeedant activities of these oxazine derivatives have been evaluated using the $4^{\text {th }}$ instar larvae Acheoa Janata $L$ by castor leaf disc method. The compounds 12-17 highly halogen substituted shows significant insect antifeedant activities.

\section{ACKNOWLEDGMENT}

The authors thank DST NMR facility, Department of Chemistry, Annamalai University, Annamalainagar608002, India, for recording NMR spectra of all compounds.

\section{Referenes}

[1] Thirunarayanan G., Surya S., Srinivasan S., Vanangamudi G., Sathiyendiran V., Spectrochim. Acta (A) 75 (2010) 152-156.

[2] Thirunarayanan G., Q-Science connect. 2013. DOI: http://dx.doi.org/ 10.5339/ connect. 2013.6, 7.

[3] Howard Miles D., Maria Ly A., Randle S. A., Hedin P. A., Burks M. L., J. Agric. Food Chem. 35 (1987) 794-797.

[4] Omara S., et al., J. Stored Prod. Res. 43 (2007) 92.

[5] Ebadollahi A., Biharean Biologist 5 (1) (2011) 8-10.

[6] Wellsow J., Grayer R. J., Veitch N. C., Kokubun T., Lelli R., Kite, G. C., Simmonds M. S., J. Phytochem. 67 (2006) 1818-1825.

[7] Miranda C. L., Aponso G. L., Stevens J. F., Deinzer M. L., Buhler D. R., J. Agric. Food Chem. 48 (2000) 3876-3884 
[8] Kim S. I., Park C., Ohh M. H., Cho H. C., Ahn, Y. J., J. Stored Prod. Res. 39 (2003) 11 19.

[9] Hiiesaar K., Švilponis E., Metspalu L., Jõgar K., Mänd M., Luik A., Karise R., Agronomy Res. 7 (2009) 251-256.

[10] Niemeyer H. M., J. Agric. Food Chem. 57(5) (2009) 1677-1696.

[11] Bhagat R. B., Kulkarni D. K., Ann. Biol. Res. 3(6) (2012) 2911-2916.

[12] Yasui H., JARQ 36(1) (2002) 25-30.

[13] Szczepanik M., Obara R., Szumny A., Gabryś B., Halarewicz-Pacan A., Nawrot J., Wawrzeńczyk C., J. Agric. Food Chem. 53(15) (2005) 5905-5910.

[14] Sunnerheim K, Nordqvist A, Nordlander G., Borg-Karlson A. K., Rickard Unelius C., Bohman B., Nordenhem H., Hellqvist C. and Karlén A., J. Agric. Food Chem. 55(23) (2007) 9365-9372.

[15] Sreelatha T., Hymavathi A., Suresh Babu K., Madhusudana Murthy J., Usha Rani P., Madhusudana Rao J., J. Agric. Food Chem. 57(14) (2009) 6090-6094.

[16] González-Coloma A., Gutiérrez C., Cabrera R., Reina M., J. Agric. Food Chem. 45(3) (1997) 946-950.

[17] Thirunarayanan G., J. Indian Chem. Soc. 85 (2008) 447-451.

[18] Morimoto M., Matsuda K., Ohta Y., Ihara T., Komai K., J. Agric. Food Chem. 52(15) (2004) 4737-4739.

[19] Thirunarayanan G., Mayavel P., Thirumurthy K., Vanangamudi G., Lakshmanan K., Sekar K. G., Int. J. Chem. 1(2) (2012) 166-172.

[20] Nalwar Y. S., Sayyed M. A., Mokle S. S., Zanwar P, R., Vibhute Y. B., World J. Chem. 4 (2009) 123.

[21] Dasharathi D., Netaji R., Basheer M. A., Vibhute Y. B., Ultra Science 17 (2005) 89.

[22] Dethler V. G., Chemical insect attractants and Repellants. Blackistan, Philadeciphia, pp. 210. (1947).

[23] Arulkumaran R., Vijayakumar S., Sundararajan R., Sakthinathan S. P., Kamalakkannan D., Suresh R., Ranganathan K., Vanangamudi G., Thirunarayanan G., International Letters of Chemistry, Physics and Astronomy 4 (2012) 17-38.

[24] K. Ranganathan, R. Suresh, D. Kamalakkannan, R. Arulkumaran, R. Sundararajan, S. P. Sakthinathan, S. Vijayakumar, G. Vanangamudi, K. Thirumurthy, P. Mayavel, G. Thirunarayanan, International Letters of Chemistry, Physics and Astronomy 4 (2012) 66-75.

[25] R. Arulkumaran, S. Vijayakumar, R. Sundararajan, S. P. Sakthinathan, D. Kamalakkannan, R. Suresh, K. Ranganathan, P. R. Rajakumar, G. Vanangamudi, G. Thirunarayanan, International Letters of Chemistry, Physics and Astronomy 5 (2013) 21-38.

[26] S. Vijayakumar, R. Arulkumaran, R. Sundararajan, S. P. Sakthinathan, R. Suresh, D. Kamalakkannan, K. Ranganathan, K. Sathiyamoorthy, V. Mala, G. Vanangamudi, G. Thirunarayanan, International Letters of Chemistry, Physics and Astronomy 9(1) (2013) 68-86. 
[27] Thirunarayanan G., Sekar K. G., International Letters of Chemistry, Physics and Astronomy 10 (2013) 18-34.

[28] R. Sundararajan, R. Arulkumaran, S. Vijayakumar, D. Kamalakkannan, R. Suresh, S. John Joseph, K. Ranganathan, S. P. Sakthinathan, G. Vanangamudi, G. Thirunarayanan, International Letters of Chemistry, Physics and Astronomy 1 (2014) 67-73. 